\title{
Enhanced timing accuracy for Cluster data
}

\author{
K. H. Yearby, S. N. Walker, and M. A. Balikhin \\ University of Sheffield, Department of Automatic Control and Systems Engineering, Mappin Street, Sheffield, S1 3JD, UK \\ Correspondence to: K. H. Yearby (k.h.yearby@sheffield.ac.uk)
}

Received: 14 June 2013 - Published in Geosci. Instrum. Method. Data Syst. Discuss.: 9 August 2013

Revised: 20 November 2013 - Accepted: 21 November 2013 - Published: 19 December 2013

\begin{abstract}
The standard timing accuracy for the Cluster mission is $\pm 2 \mathrm{~ms}$. However for inter-spacecraft comparisons of waveform data, a much higher accuracy is needed - for example a timing error of $1 \mathrm{~ms}$ results in a phase error of $65^{\circ}$ for a signal at $180 \mathrm{~Hz}$. Most Cluster data are recorded on an onboard solid state recorder and time-stamped using an onboard clock which is calibrated to coordinated universal time (UTC). Until recently, the error of this onboard clock was allowed to increase to the $\pm 2 \mathrm{~ms}$ limit before a new calibration was applied. However, the timing error for real-time data is estimated to be only $\sim 11 \mu$ s, so these data may be used to prepare a time correction data set which allows the standard timing accuracy to be improved considerably. This paper describes the details of the preparation and validation of this data set. Two independent source data sets are used: telemetry to European Space Agency (ESA) ground stations supporting the main operations of the Cluster spacecraft, and the real-time telemetry to the NASA Deep Space Network (DSN) stations supporting the Wide-Band Plasma Wave Investigation.
\end{abstract}

\section{Introduction}

The Spatio-Temporal Analysis of Field Fluctuations (STAFF) and Electric Field and Wave (EFW) experiments, forming part of the Wave Experiment Consortium (WEC) on the Cluster spacecraft, include magnetic and electric field waveform data at frequencies up to $180 \mathrm{~Hz}$ in the high bit rate mode (Cornilleau-Wehrlin et al., 199; Gustafsson et al., 1997; Pedersen et al., 1997). Inter-spacecraft comparison of these data, for example to estimate wave properties by $k$ filtering or phase differencing, requires better than the standard $2 \mathrm{~ms}$ timing accuracy.
The timing accuracy required is discussed by Pincon and Motschmann (1998), and a series of simulations performed by Pincon and Lefeuvre (1992). These suggest the phase error should ideally be less than $5^{\circ}$, and that the fit with the model becomes very poor for an error of $30^{\circ}$ or above. At a frequency of $180 \mathrm{~Hz}$, these phase errors correspond to a timing accuracy of $77 \mu \mathrm{s}$, and $460 \mu$ s respectively.

An alternative estimate of the timing accuracy required may be derived from the propagation speed of the waves and the accuracy of the spacecraft position. For example a whistler mode wave in the magnetosheath with a frequency of $150 \mathrm{~Hz}$ propagates at around $3 \times 10^{6} \mathrm{~ms}^{-1}$. The maximum error in the spacecraft position is $5 \mathrm{~km}$, so the timing error should be much less than $1.67 \mathrm{~ms}$ to avoid contributing to errors in the estimated wave properties. Timing accuracy is more important at small spacecraft separations as coherence between the measurements is maintained at higher frequencies.

The Wide-Band Data (WBD) Plasma Wave Investigation (Gurnett et al., 1997) measures waves up to $577 \mathrm{kHz}$. At these frequencies (above the local plasma frequency) waves can propagate at close to the velocity of light $\left(3 \times 10^{8} \mathrm{~ms}^{-1}\right)$, so a timing accuracy better than $15 \mu$ s is ideally needed.

This need for high timing accuracy was recognised during the proposal phase of the Cluster mission, but not accepted by the agency as a formal requirement. Instead the guaranteed timing accuracy was $2 \mathrm{~ms}$, but with an expectation that considerably better accuracy could be achieved in practice. In the worst case, a $2 \mathrm{~ms}$ accuracy corresponds to a $4 \mathrm{~ms}$ error between spacecraft, which is $260^{\circ}$ of phase at $180 \mathrm{~Hz}$. In the normal bit rate, for which the maximum signal frequency is $10 \mathrm{~Hz}$, the maximum phase error would be $12^{\circ}$.

To understand how higher accuracy can be achieved, it is first necessary to explain how the standard time-stamping of Cluster data is performed. Most Cluster data are not acquired 
from the spacecraft in real time, but are recorded on an onboard solid state recorder. All data released to experimenters (both recorded on board and acquired in real time) are timestamped using an onboard time (OBT), which is calibrated to UTC using a time correlation process.

Experiment data are not time-stamped directly. Timing is coordinated by means of a reference pulse distributed to the experiments by the spacecraft onboard data handling system $(\mathrm{OBDH})$. It is the responsibility of each experiment to determine the time of data sampling relative to this reference pulse and include this information as part of the experiment data. Each packet of experiment data acquired by the $\mathrm{OBDH}$ is then stamped with the time of the reference pulse.

Until recently, the error between OBT and UTC was allowed to increase to the $\pm 2 \mathrm{~ms}$ limit before a new calibration was applied. This typically occurred around once per month. The time correlation process uses the real-time data, which are time-stamped by the ground stations with the Earth receive time. By subtracting various delays, including the propagation time from spacecraft to ground, the spacecraft event time (SCET) is obtained. The total error for real-time data is estimated to be $\sim 11 \mu$ s, determined by adding various error sources, the main contributions being uncertainty in the spacecraft position and ground station clock drift (ESOC report CL-ESC-TN-0006, personal communication, 1992).

During each real-time pass, the difference (DIFF) between the SCET measured by the onboard clock and that derived from the Earth receive time is monitored. When the difference exceeded $2 \mathrm{~ms}$, a new time correlation was performed. This process is described in detail in the European Space Operations Centre (ESOC) report CL-OPS-TN-1004-OPS-OPC provided to the authors as a private communication, but this should be available from the Cluster Science Archive in due course.

The DIFF measurements can be used to apply a correction to the onboard clock to bring its accuracy close to that of the real-time data. The DIFF is slowly varying, so a simple linear interpolation can be used to estimate the DIFF between real-time passes. An independent set of DIFF measurements are also available during real-time data acquisition for the WBD experiment from the ground stations of the NASA Deep Space Network (DSN) and Panska Ves. An example of the variation in the DIFF parameter is shown in Fig. 1.

Detailed analysis of the time stamps has revealed that a second correction is needed. When data are recorded on board, the OBT recorded in the data packets is not precisely correlated with the timing reference pulse distributed to the experiments but may be subject to an offset which is fixed for each period of the same telemetry mode. This term is called OFFSET and specifies the difference between the time of the reference pulse and the OBT recorded in the data packets. It applies only to data recorded on board, and is zero for real-time data. OFFSET normally ranges $\pm 180 \mu$ s for normal mode data, and $\pm 30 \mu$ s in burst modes.

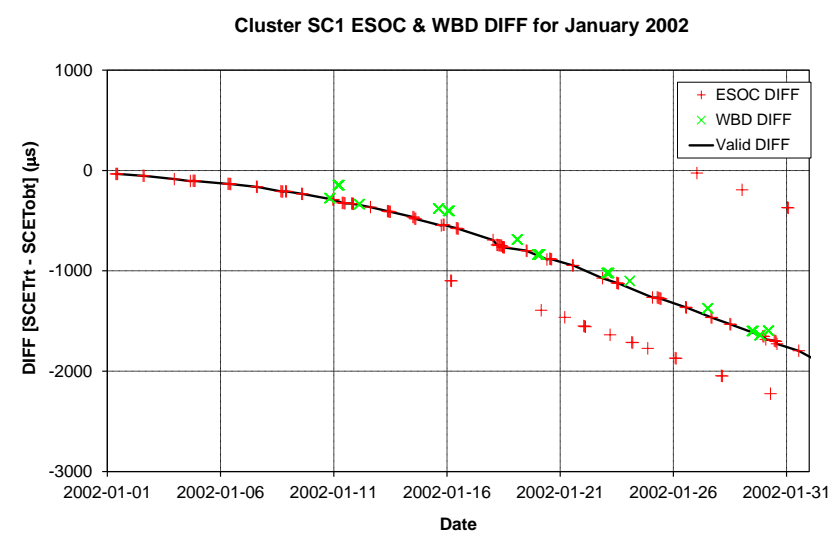

Fig. 1. Plot of the DIFF values measured by ESOC (+) and WBD $(\times)$. The solid line represents the assumed correct value used to prepare the time corrections. The "DIFF" (the difference between the ground receive time and the onboard time) is measured during every ground station contact. However some measurements are subject to errors, so a validation process must be applied.

The DIFF and OFFSET parameters together form the Time Correction (TCOR) data set produced by the Digital Wave Processor (DWP) team and available from the Cluster Science Archive (CSA) (Yearby et al., 2010; Woolliscroft et al., 1997).

From 23 November 2007, a new time correlation procedure has been adopted at ESOC. A time correlation is now performed during every nominal pass, instead of waiting until the DIFF reaches $2 \mathrm{~ms}$. As a result the value of DIFF is usually less than $20 \mu \mathrm{s}$, but this is not guaranteed. While a correction for the OFFSET term is in principle still needed, for burst mode data (when the most accurate timing is needed), this is less than $30 \mu \mathrm{s}$, so without correction the overall timing will usually be accurate to $\pm 50 \mu$ s. This corresponds to $100 \mu$ s between spacecraft, which is sufficient for STAFF and EFW data up to $180 \mathrm{~Hz}$. However, it will still be possible to use the time correction data set when the highest possible accuracy is needed.

\section{Production}

\subsection{DIFF pre-November 2007}

The DWP team use the time difference measured by both ESOC and WBD to prepare the time correction data set. Both ESOC and WBD timing are sometimes subject to errors due to ground station configuration problems, so these data are subject to a manual validation process, illustrated in Fig. 1. It is observed that most of the points fall close to one line. A smooth curve is drawn through these points and taken to be the correct value. The result is a set of DIFF measurements valid at specific points in time (during European Space Agency (ESA) or DSN/Panska Ves ground station passes). 
Recalibrated ESOC and WBD DIFF measurements, Cluster C2, 2008

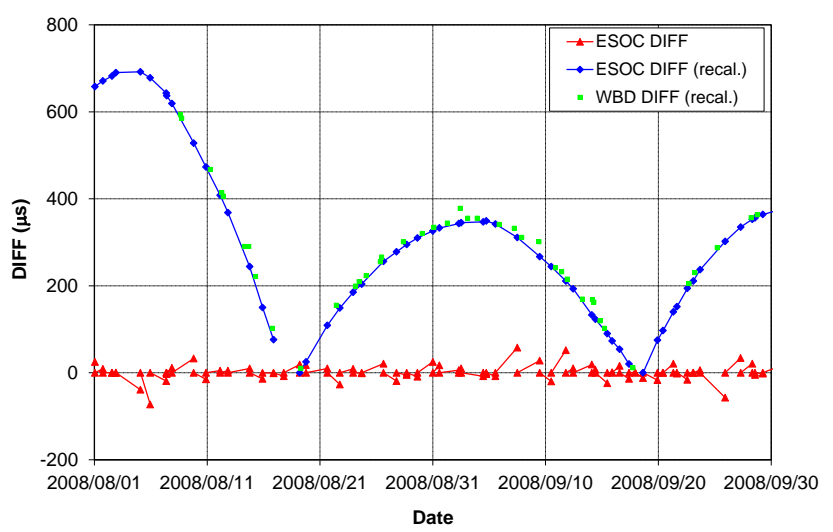

Fig. 2. Recalibrated DIFF measurements after November 2007. When time calibrations are performed every pass, the DIFF values are small and give no indication of the long-term behaviour of the onboard clock. The recalibrated DIFF has a parabolic form similar to that seen in Fig. 1.

To obtain DIFF between measurements, a linear interpolation is normally used.

\subsection{DIFF post-November 2007}

Since 23 November 2007, a time calibration has been performed during every nominal pass. This process resets the DIFF to zero, so the trends shown in Fig. 1 are no longer seen, and it is difficult to validate the data directly. Instead the DIFF is recalibrated relative to virtual time calibrations performed every 30 days. This allows any discontinuities to be easily seen (Fig. 2). This recalibrated DIFF is used only for validation purposes.

\subsection{WBD DIFF}

The DIFF values are calculated using the OBT and ground receive time fields in the WBD "level 1" data released by the University of Iowa. The values are then compared against a linear interpolation between the two nearest ESOC DIFF measurements. The difference between the WBD DIFF and interpolated ESOC DIFF is termed "delta". Typical results are shown in Fig. 3a, and are normally in the range 0 to $50 \mu \mathrm{s}$. However, when the data are plotted against the ground station antenna number, as shown in Fig. 3b, then we see that each antenna has a range of delta values covering only $20 \mu \mathrm{s}$. The mean offset for each antenna can be subtracted to give recalibrated WBD DIFF values with a mean as close as possible to the ESOC ones. In practice, just three separate offset values are used: one for antenna 46, one antennae 80 and 81 , and one for all others. In each case the same offset values apply to all Cluster spacecraft. (a)
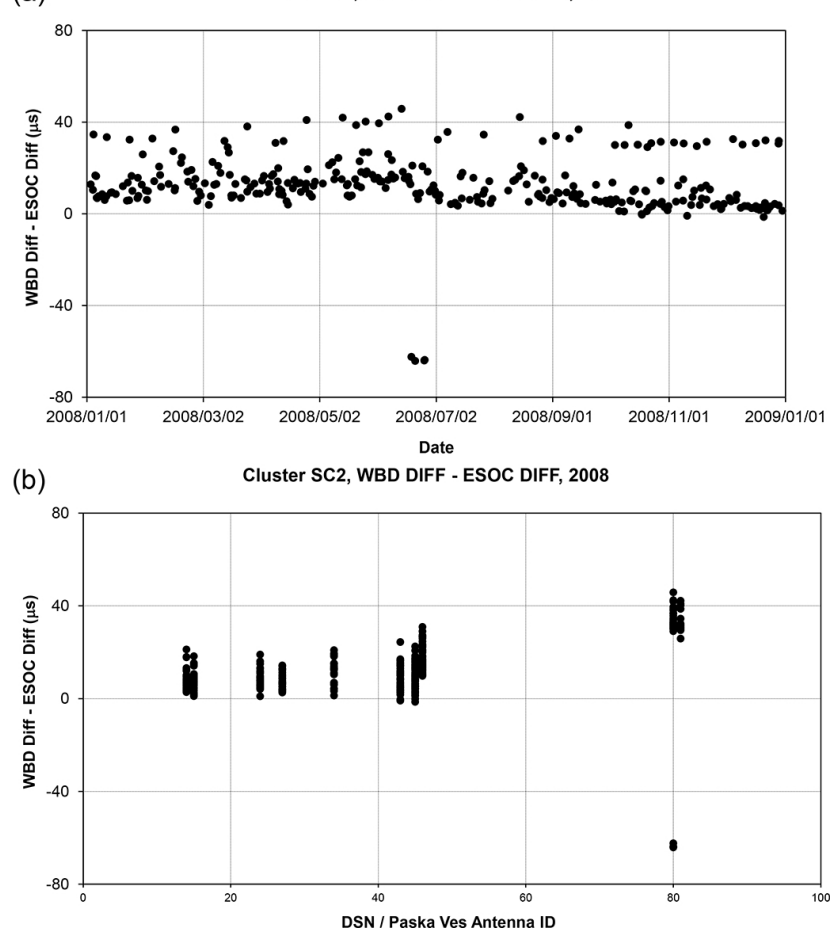

Fig. 3. Comparison of DIFF measured during WBD (DSN/Panska Ves) and ESOC ground station contacts. WBD-ESOC DIFF versus date shows a bias, usually small and positive (a). Plotting the same data versus antenna ID shows antennae 46, 80 and 81 have a different bias to the others (b). Data for antenna 80, near the end of June, are anomalous due to a timing problem at the Panska Ves station.

\subsection{OFFSET}

The OFFSET term is determined by the DWP team by analysis of the time stamps of the housekeeping telemetry. The OBT is maintained by counting the cycles of a $2^{20} \mathrm{~Hz}$ clock. The count is padded on the right-hand side with four zero bits to form the 56 bit OBT value. Taken as a whole this count represents the cycles of a virtual $2^{24} \mathrm{~Hz}$ clock. This raw OBT value is not included in the data released to experimenters. Rather, it is converted to UTC by applying the current time calibration. The first stage of the OFFSET analysis is to obtain (approximately) the raw OBT by inverting the time calibration.

The telemetry format generator issues the timing reference pulse every $5.152222 \mathrm{~s}$ or exactly $42207 \times 2^{11}$ OBT counts. Therefore OBT modulo $42207 \times 2^{11}$ should be constant. We call this quantity OBTM, and the typical variation is shown in Fig. 4. OBTM changes at every telemetry mode change, but comparison with independent regular events reveals that there is usually no change in the regular occurrence of the timing reference pulse. Furthermore OBTM usually returns to the same value for each period of real-time telemetry. Therefore we conclude that when data are recorded on board, 
there must be a variable offset between the time at which the reference pulse is issued and the OBT recorded in the data. We assume that this offset is zero for real-time data, although any constant value, the same for all spacecraft, would not affect inter-spacecraft timing accuracy. The deviation of OBTM from the real-time value is the OFFSET parameter.

A difficulty arises when there is a change in the regular occurrence of the timing reference pulse. Small changes of $122 \mu$ s (2048 OBT counts) sometimes occur when the OBDH mode changes. This is first apparent when the OBTM during a period of real-time telemetry differs from that seen previously. It is then necessary to determine when the change occurred. This is done by analysing the times of independent regular events measured relative to the reference pulse. Two independent events are currently used: the internal master clock pulse of the WEC and the Sun reference pulse.

Finally, the DIFF and OFFSET values are merged to form the TCOR data set, which is available from the Cluster Science Archive (CSA). This data set has a pair of records for each period of constant OFFSET. Linear interpolation of the original DIFF values is used to obtain values at the times of OFFSET changes. The CSA may be accessed at http://www.rssd.esa.int/index.php?project= CLUSTER\&page=Access_to_CSA.

\section{Validation}

Whilst the time correction process seems straightforward, there are a number of difficulties that can arise even after the initial DIFF measurements have been validated.

\subsection{Validity of original OBT-UTC calibration}

The initial time calibration is performed by ESOC and recorded in the TCAL files that are included with the experiment raw data. Experimenters do not usually need to refer to these files as the calibrations are already applied. They are however essential for the purpose of making enhanced accuracy timing as the corrections applied are relative to the original calibration. Initially this caused a difficulty, as prior to November 2007 the period to which each calibration applied was not known precisely. This occurred because whenever a new calibration was determined it was applied to all data received on the ground from that point onwards, including data that may have been acquired on board up to 2 days before when a previous calibration was still active.

Use of the wrong time calibration becomes apparent during the process of estimating the OFFSET parameter. Instead of the quantity OBTM being constant with step changes, it shows a continuous linear change. Such data are rejected during processing, and are the main reason for the periods where time corrections are not available during this early part of the mission.
After November 2007 the process at ESOC was changed so time calibrations were applied strictly according to their period of validity using the time that data were originally acquired on board.

\subsection{Interruptions to the onboard clock}

Onboard anomalies can cause a switchover from the main to the redundant data handling system, or an eclipse can cause the loss of all power on the spacecraft (there is now little or no battery capacity left). Such events cause an interruption to the onboard clock requiring a completely new time calibration. Furthermore, after such an event it may take several days for the stability of the clock to return to normal. This means the normal linear interpolation of DIFF values may be impossible to perform or have reduced accuracy.

\subsection{Changes to the reference pulse timing}

As noted in Sect. 2.4 above, changes may occur in the regular occurrence of the timing reference pulse. We attempt to identify these by reference to two independent regular events: the internal master clock pulse of the WEC experiment and the Sun reference pulse. Neither method is completely satisfactory.

The WEC master clock is $900 \mathrm{~Hz}$ and derived from a basic crystal oscillator with a stability of about 1 part in $10^{6}$. The time of the clock pulses is measured using a software timer. This is normally done on every timing reference pulse (every $5.152222 \mathrm{~s}$ ) and has an accuracy of a few microseconds. Sometimes however the measurement is interrupted by other processes, so it is not available. This can make it difficult to identify exactly when any change in the reference pulse timing has occurred.

The Sun reference pulse is generated when the spacecraft spin brings the Sun sensor in alignment with the Sun direction. This process is normally remarkably stable in the short term (to within a few microseconds in the $4 \mathrm{~s}$ spin) but is upset following a manoeuvre when oscillations in the spin period can continue for several hours. The Sun reference pulse is currently used during the production process, with the WEC master clock used for validation.

Routine validation of the TCOR data set is performed by computing the difference between the time stamps of successive telemetry formats. The maximum, minimum, mean, and standard deviation from the nominal value are computed for each $24 \mathrm{~h}$ period. The nominal value is $5.152222 \mathrm{~s}$, but the validation process allows for the $122 \mu$ sumps in the regular occurrence of the timing reference pulse that are known to sometimes occur. Differences larger than about $3 \mu$ indicate an error in the production. 
Cluster SC3, 2007/10/24-25, OFFSET

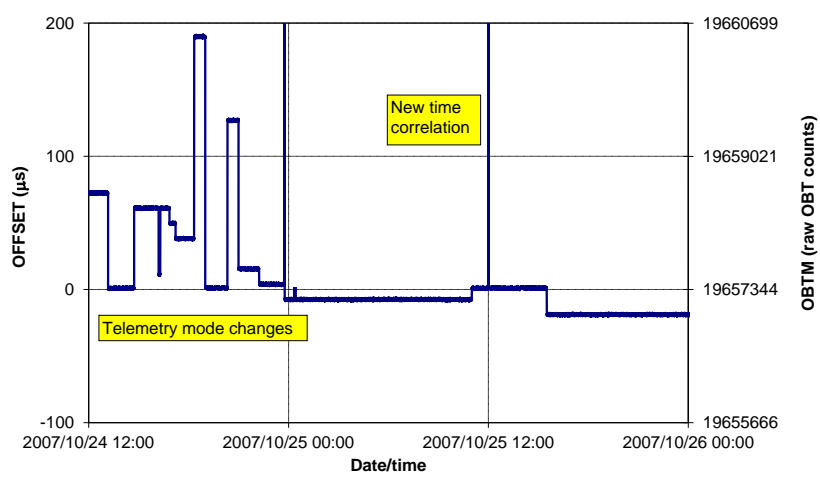

Fig. 4. OFFSET specifies the difference between the time of the reference pulse (VCO reset pulse) and the onboard time stamp recorded in the data packets. It applies only to data recorded on board, changes at every telemetry mode change, and is zero for real-time data. OFFSET normally ranges $\pm 180 \mu$ s for normal mode data, and $\pm 30 \mu$ s in burst modes. It is derived from analysis of the raw onboard time modulo $42207 \times 2^{11}$ (termed OBTM).

\section{Caveats}

Users of the TCOR data set should be aware of its limitations. Most importantly, time corrections are not available at all times. Coverage is typically around $90 \%$. Applications requiring accurate timing should confirm that TCOR data are available at the relevant time.

Time corrections are provided at the start and end of each segment (a time interval with no telemetry mode changes). These segments have a duration from a minimum of one minute to a maximum of about $27 \mathrm{~h}$ (half an orbit). Time corrections at other times may be obtained by interpolation subject to certain rules. The OFFSET term is constant throughout each segment. When the OFFSET values before and after the required time are different, then OFFSET is not available at that time. No interpolation between different OFFSET values is allowed. The DIFF may be obtained by linear interpolation of the DIFF values at the beginning and end of the current segment.

\section{Application}

For WEC data, the time corrections may be applied using the latest version of the Telemetry Extraction and Decommutation (TED) sofware package (see Yearby et al., 2010). This is done automatically once TED and the TCOR files are correctly installed. By default, TED 2.5 produces decommutated science data files with all time corrections applied. A new bit in the packet header diagnostic word (HKTCOR_CORRECTED) will be set when time corrections have been successfully applied. This bit should be checked by applications requiring accurate timing.

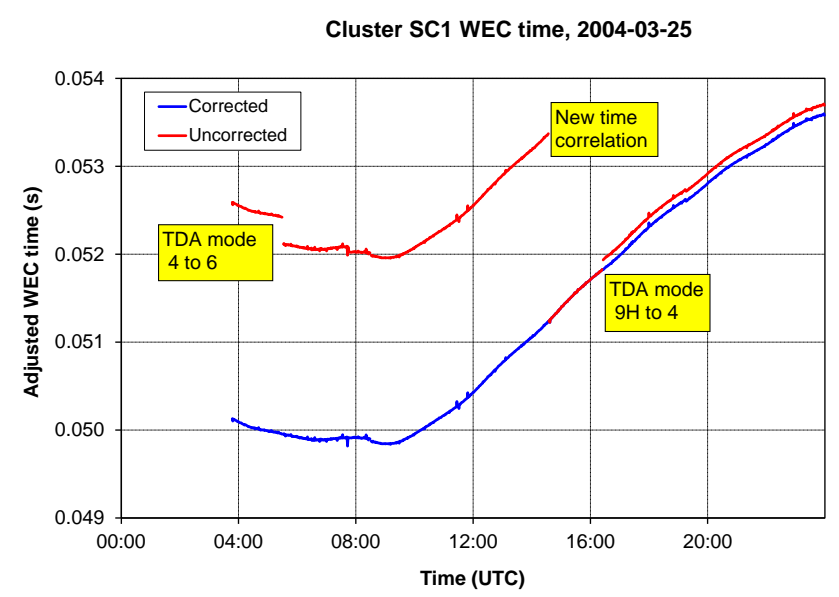

Fig. 5. Final validation of time corrections. The chart shows WEC data time stamps modulo $0.1 \mathrm{~s}$, and are adjusted for the average rate of drift of the WEC clock. The uncorrected time shows jumps due to the change in OFFSET at telemetry data acquisition (TDA) mode changes, and due to change in DIFF at a new time correlation. These jumps are removed when the corrections are applied.

The result of applying time corrections is illustrated in Fig. 5. The uncorrected time shows jumps due to the change in OFFSET at telemetry data acquisition (TDA) mode changes, and due to change in DIFF at a new time correlation. These jumps are removed when the corrections are applied.

It is also possible to apply time corrections to the raw telemetry data or to calibrated high-level science data, provided that microsecond level precision is maintained during the processing of the data. The basic method is to obtain the DIFF and OFFSET at the required time, and add these to the data time tag.

The TCOR data set is applicable to all data acquired from the Cluster spacecraft via ESA ground stations. The TCOR data set itself is generally not used for creation of the epoch variable in the WBD CDF/CEF data files archived at the CSA. This is because WBD data acquired via DSN or Panska Ves ground stations already have sufficient timing accuracy. However, on rare occasions the TCOR production process identifies apparent errors in the time calibration of these ground stations. For these cases, the WBD team will use the TCOR data set to correct the onboard time supplied in the WBD transfer frame to obtain the epoch variable. The timing accuracy in these cases will be comparable to that attainable via DSN or Panska Ves ground stations.

\section{Conclusions}

The standard $2 \mathrm{~ms}$ timing accuracy for the Cluster mission is not sufficient for inter-spacecraft comparisons of high bit rate waveform data. The timing accuracy may be enhanced 
to the order of $20 \mu$ s by applying corrections from the TCOR data set in the Cluster Science Archive.

For future missions, the required timing accuracy should, if possible, be accepted as a formal specification. If this is not practical, then procedures should be adopted to get the best accuracy reasonably achievable rather than just meeting the specification. The change in procedure at ESOC from November 2007 (a new time correlation during every nominal pass) dramatically improved the basic timing accuracy achieved from $2 \mathrm{~ms}$ to around $50 \mu \mathrm{s}$.

Acknowledgements. The authors thank colleagues at ESOC and the University of Iowa for providing the source data for the time correction data set. The current work and the Cluster mission were funded by the European Space Agency.

Edited by: C.-P. Escoubet

\section{References}

Cornilleau-Wehrlin, N., Chauveau, P., Louis, S., Meyer, A., Nappa, J. M., Perraut, S., Rezeau, L., Robert, P., Roux, A., de Villedary, C., de Conchy Y., Friel, L., Harvey, C. C., Hubert, D., Lacombe, C., Manning, R., Wouters, F., Lefeuvre, F., Parrot, M., Pinçon, J. L., Poirier, B., Kofman, W., and Louarn, Ph.: The CLUSTER Spatio-Temporal Analysis of Field Fluctuations (STAFF) Experiment, Space Sci. Rev., 79, 107-136, 1997.

Gurnett, D. A., Huff, R. L., and Kirchner, D. L.: The wide-band plasma wave investigation, Space Sci. Rev., 79, 195-208, 1997.

Gustafsson, G., Bostrom, R., Holback, B., Holmgren, G., Lundgren, A., Stasiewicz, K., Ahlen, L., Mozer, F. S., Pankow, D., Harvey, P., Berg, P., Ulrich, R., Pedersen, A., Schmidt, R., Butler, A., Fransen, A. W. C., Klinge, D., Thomsen, M., Falthammar, C. G., Lindqvist, P. A., Christenson, S., Holtet, J., Lybekk, B., Sten, T. A., Tanskanen, P., Lappalainen, K., and Wygant, J.: The electric field and wave experiment for the Cluster mission, Space Sci. Rev., 79, 137-156, 1997.
Pedersen, A., Cornilleau-Wehrlin, N., de la Porte, B., Roux, A., Bouabdellah, A., Décréau, P. M. E., Lefeuvre, F., Sené, F. X., Gurnett, D., Huff, R., Gustafsson, G., Holmgren, G., Woolliscroft, L. J. C., Thompson, J. A., and Davies, P. H. N.: The Wave Experiment Consortium (WEC), Space Sci. Rev., 79, 93-106, 1997.

Pincon, J.-L. and Lefeuvre, F., The application of the generalized Capon method to the analysis of a turbulent field in space plasma: experimental constraints, J. Atmos. Terr. Phys., 54, 1237-1247, 1992.

Pincon, J.-L. and Motschmann, U.: Multi-Spacecraft Filtering: General Framework, in: Analysis Methods for Multi-Spacecraft Data, Paschmann G. and Daly, P. W., ESA Publications Division, Noordwijk, the Netherlands, 65-78, 1998.

Woolliscroft, L. J. C., Alleyne, H. St. C., Dunford, C. M., Sumner, A., Thompson, J. A., Walker, S. N., Yearby, K. H., Buckley, A., Chapman, S., Gough, M. P., and the DWP Co-investigators: The digital wave processing experiment on Cluster, Space Sci. Rev., 79, 209-231, 1997.

Yearby, K. H., Alleyne, H. St. C., Walker, S. N., Bates, I., Gough, M. P., Buckley, A., and Carozzi, T. D.: Digital Wave Processor Products in the Cluster Active Archive, in: The Cluster Active Archive, Studying the Earth's Space Plasma Environment, edited by: Laakso, H., Taylor, M. G. T. T., and Escoubet, C. P., Astrophysics and Space Science Proceedings, Berlin: Springer, 73-82, 2010 . 